\title{
Rabbit Aneurysm Model Mediated by the Application of Elastase
}

\author{
Kuri SASAKI, Hiroshi UjIIE, Takashi HIGA, Tomokatsu HORI, \\ Noriko SHINYA*, and Takanori UCHIDA*
}

\begin{abstract}
Department of Neurosurgery, Neurological Institute, Tokyo Women's Medical University, Tokyo; ${ }^{*}$ The Chemo-Sero-Therapeutic Research Institute, Kumamoto
\end{abstract}

\begin{abstract}
The concentrations and application methods of elastase in the rabbit aneurysm model were optimized to control the initiation of aneurysms and to cause rupture in a stepwise, controlled fashion. The common carotid artery of male Japanese albino rabbits was exposed. No aneurysm was generated if the adventitia was not dissected. After gentle removal of the adventitia, a two-fold dilution series of elastase was applied to the lesion and observed over a period of 2 hours. Various stages of aneurysmal lesions, from spindle-shaped enlargement to rupture, were produced in proportion to the elastase concentration. Application of elastase stock solution (5 U/mg of type I porcine pancreatic elastase) resulted in rupture within 30 minutes in all six animals. Elastase 1:2 solutions caused oozing in all animals, but subsequent rupture in only three of six animals. Histological examination found serious destruction of the internal elastic lamina and media, with expansion of the very thin wall. Elastase 1:4 to 1:16 solutions caused spindle-like distention of the entire artery and the development of tortuosity at the lesion. Elastase 1:32 or weaker solutions caused only localized dilatations. Overall, the destruction of the tunica media became less severe with decreased elastase concentration. Furthermore, the bursting pressure of the aneurysms decreased with increasing elastase concentrations. In particular, aneurysms produced by the elastase 1:2 solution ruptured at less than $150 \mathrm{~mm} \mathrm{Hg}$, whereas aneurysms induced by the elastase 1:4 or weaker solutions did not rupture within the physiological range of blood pressure. The present aneurysm model requires shorter preparation time and enables accurate control of aneurysm development and rupture.
\end{abstract}

Key words: aneurysm, experimental aneurysm, elastase, subarachnoid hemorrhage

\section{Introduction}

Rupture of a cerebral aneurysm leads to the catastrophic consequences of subarachnoid hemorrhage, so unruptured aneurysms are now frequently treated in Japan. ${ }^{20,24)}$ However, neither the mechanism of development and growth nor the process leading to rupture of cerebral aneurysms has been clarified. Consequently, the clinical problems of how to prevent the development of cerebral aneurysms and how to predict rupture remain unresolved..$^{27,28)}$ Various animal experimental models have been established to elucidate the mechanisms of initiation, growth, and rupture of cerebral aneurysms. ${ }^{1,3,6,11-15,18,19,23,28)}$ A widely used model consists of aneurysms which are surgically created by suturing a vein pouch onto an arterial bifurcation or the artery. ${ }^{11,23)}$ This model is reproducible and easy to implement, but the surgically created aneurysms are histologically different from natural aneurysms and can be used only for limited purposes. Aneurysms that histologically resemble human cerebral aneurysms can be produced by the simultaneous loading of hemodynamic stress and inducing the vulnerability of blood vessel walls. ${ }^{13-15)}$ This model indicates that hemodynamic stress and vulnerability of the blood vessel wall are both important in the development of aneurysms. However, this experimental model may be used only for limited purposes because the timing

Received May 20, 2003; Accepted April 13, 2004

Author's present address: K. Sasaki, M.D., Department of Neurosurgery, Tokyo Metropolitan Ohkubo Hospital, Tokyo, Japan. 
and position of the aneurysms are variable.

Histological examinations of cerebral aneurysms have demonstrated extensive destruction or absence of the tunica media muscle layer, and sudden termination of the elastic laminae, as well as serious decreases in their components, elastin and collagen. ${ }^{7,15,25)}$ In addition, elastase and various matrix metalloproteinases may damage arterial walls. ${ }^{2,4,5,9,16,22)}$ The increase of elastase concentration in the plasma and the aneurysm wall in patients with cerebral aneurysms supports the hypothesis that digestion of vascular walls by such proteases is centrally involved in the development of aneurysms. ${ }^{4,10,21,26)}$ Elastase has been used to produce models of abdominal aortic aneurysm and rabbit carotid aneurysm. ${ }^{1,8)}$ In particular, a model of aneurysms at arterial bifurcations possesses histological characteristics similar to those of human saccular aneurysms, and can be developed and ruptured rapidly. ${ }^{18)}$ The features of this model are very advantageous in aneurysm research, although the development and rupture of aneurysms are not clearly reproducible.

The present study optimized the concentrations and application methods of elastase in the established experimental model ${ }^{18)}$ to enhance the ease and accuracy of aneurysm development and graded control of rupture.

\section{Materials and Methods}

\section{Induction of aneurysm model}

The common carotid artery (about $3 \mathrm{~cm}$ in length) of male Japanese albino rabbits (3-5 months old, body weight $2.8-3.2 \mathrm{~kg}$ ) was exposed under anesthesia with Nembutal $(30 \mathrm{mg} / \mathrm{kg}$, intravenous injection; Dainippon Pharmaceutical Co., Ltd., Osaka). Elastase solutions (type I, from porcine pancreas, 9.7 $\mathrm{mg}$ protein $/ \mathrm{ml}, 5 \mathrm{U} / \mathrm{mg}$; Sigma Chemical Co., St Louis, Mo., U.S.A.) were prepared by serial two-fold dilution from the stock solution from 1:1 to $1: 128$ with physiological saline. Selective removal of the adventitia was performed by a meticulous microscopic technique to produce a small, reddish bulge on the vessel wall. The adventitia should be excised gently using forceps rather than micro-scissors to avoid injury to the tunica media or tunica intima, resulting in sudden rupture and vigorous bleeding. A 1-mm ${ }^{2}$ Bemsheet (Kawamoto Corporation, Osaka) with $50 \mu \mathrm{l}$ of adsorbed elastase was applied to the normal adventitia, and to adventitia-dissected areas for 10 minutes. Microscopic observations were carried out, and the time from elastase application to rupture was measured. If the aneurysmal lesions did not rupture, observation was continued up to 2 hours after elastase application. At the termination of observation, the aneurysmal lesion was removed for histological examination by light and scanning electron microscopy. For light microscopy, the preparation was perfusion-fixed with formalin and stained by hematoxylin-eosin and elastica van Gieson. We followed the same protocol for four to six animals for each elastase concentration. For scanning electron microscopy, the tissue was fixed by perfusion with glutaraldehyde, washed with

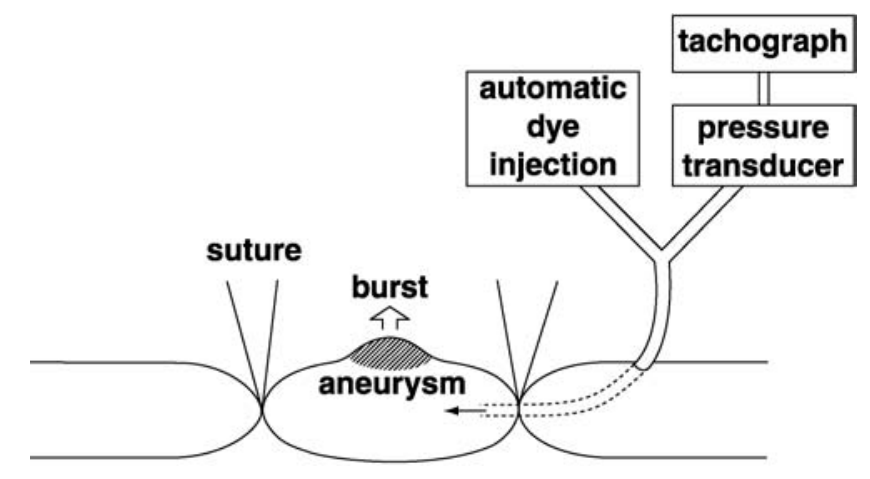

Fig. 1 Schema of the bursting test system.

Table 1 Macroscopic findings of experimental aneurysms

\begin{tabular}{|c|c|c|c|c|c|}
\hline $\begin{array}{c}\text { Concentration } \\
\text { of elastase }\end{array}$ & $\begin{array}{l}\text { No. of } \\
\text { animals }\end{array}$ & \multicolumn{2}{|c|}{ Dilatation } & \multicolumn{2}{|c|}{ Bleeding } \\
\hline $1: 2$ & 6 & 6 & 0 & 3 & 3 \\
\hline $1: 4$ & 6 & 0 & 6 & 3 & - \\
\hline $1: 8$ & 6 & 0 & 6 & - & - \\
\hline 1:64 & 4 & 4 & 0 & - & - \\
\hline $1: 128$ & 4 & 4 & 0 & - & - \\
\hline
\end{tabular}




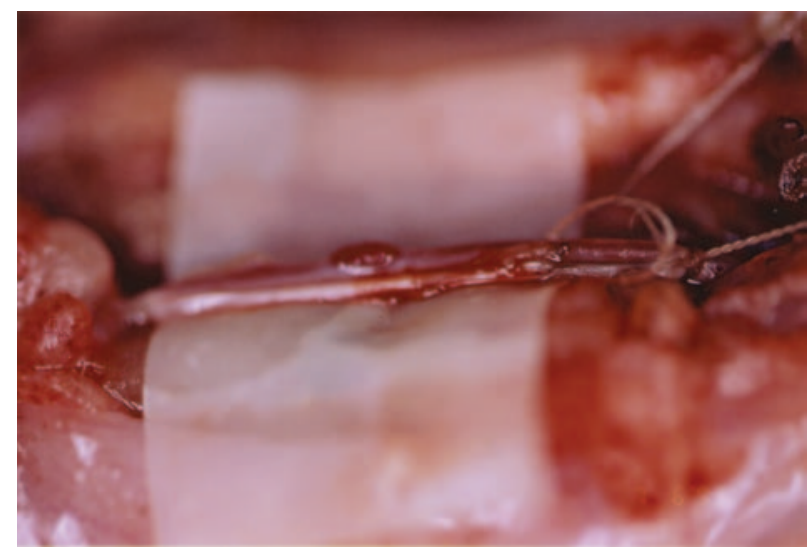

Fig. 2 Photograph of an aneurysmal lesion on the lateral wall of the common carotid artery taken 30 minutes after the adventitia was gently removed and elastase (two-fold dilution) was applied, showing localized bulging and oozing.
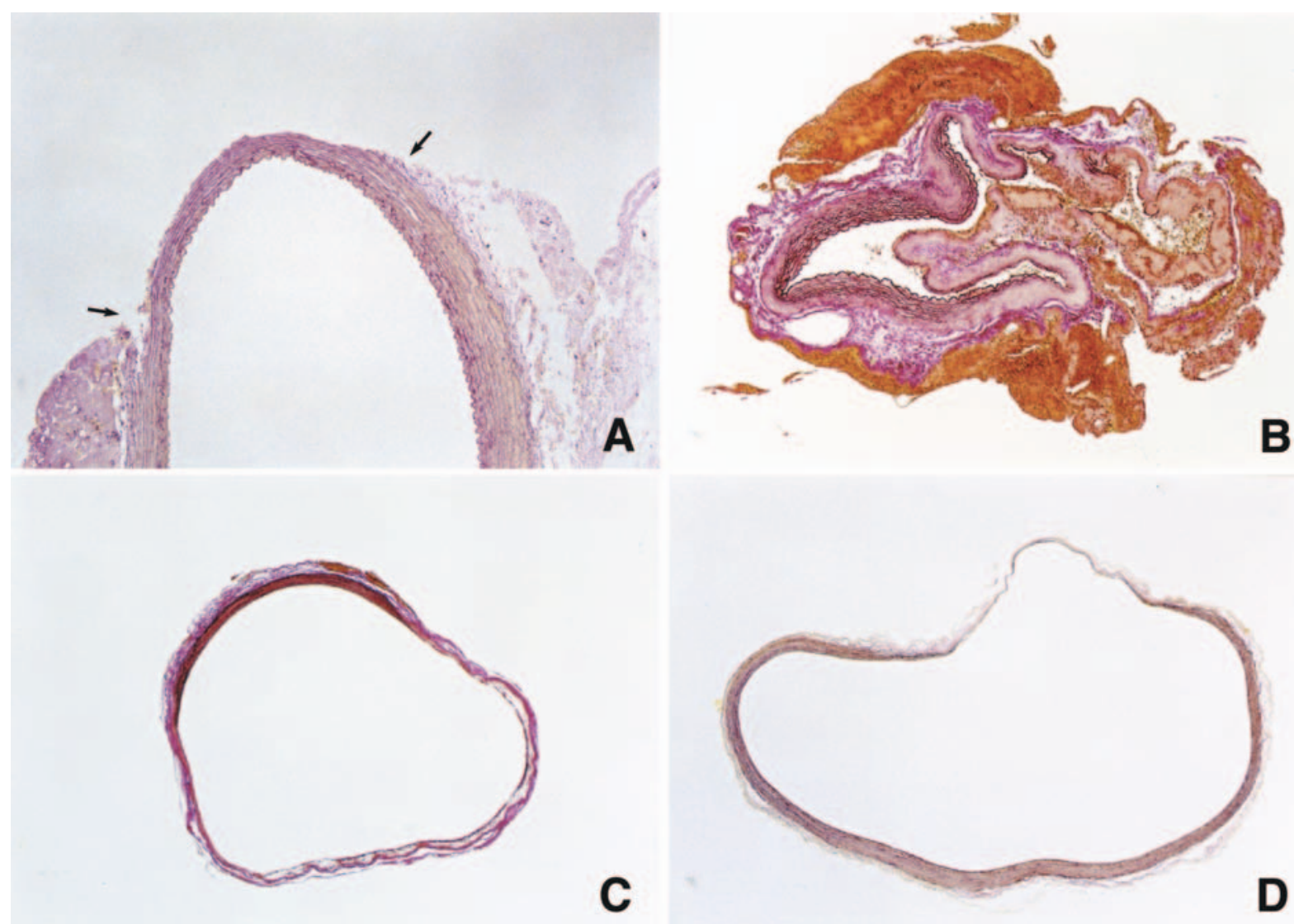

Fig. 3 Photomicrographs of an aneurysmal lesion. A: Localized excision of the adventitia and intact tunica media (arrows) is observed at the superior part of the vessel. The adventitia with the external elastic band was gently removed. B: Elastase 1:1 solution induced rupture of an aneurysmal lesion with severe destruction of the arterial wall. Enzymatically destroyed arterial wall with cellular infiltration was buried in hemorrhage. C: Elastase 1:2 solution developed localized thinning. The tunica media and elastic bands are highly eroded and almost disappeared. Medial elastolysis and necrosis of smooth muscle cells exacerbated vessel distensibility and fragility. D: Elastase 1:32 solution caused focal erosion of the tunica media. The normal structure of the tunica media is still maintained. Elastica van Gieson stain, A: $\times 25$, B: $\times 14.6, \mathrm{C}: \times 7.35, \mathrm{D}: \times 10$. 
phosphate-buffered saline $(0.1 \mathrm{M})$, refixed in osmium tetroxide, dehydrated and dried, and observed after sputter coating with platinum. This process was performed in three animals with elastase 1:2 and 1:32 solutions.

\section{Forced rupture tests}

Forced rupture tests were performed according to the reported method for the rabbit aneurysm model established by the adventitia-dissection method. ${ }^{17)}$ The common carotid artery was exposed and elastase 1:2, 1:4, 1:8, and 1:16 solutions were applied at a relatively distal site. The application period was 10 minutes for elastase 1:2 and 1:4 solutions, and 1 hour for more dilute elastase solutions. After elastase application, a 22-G catheter was inserted from $2 \mathrm{~cm}$ up to about $1 \mathrm{~cm}$ proximal to the application site, and fixed with a silk-thread ligature to prevent blood flow. A Y-connector was attached to the catheter. One end was connected through an extension tube to a $20 \mathrm{ml}$ syringe containing physiological saline mixed with Evans blue, and the other was connected to a pressure-measuring device. After washing out the blood in the arterial lumen with saline, the distal tip of the lesion was tied with silk thread. Evans blue-containing saline was injected with a pump at $0.1 \mathrm{ml} / \mathrm{min}$ until the aneurysm ruptured, and the pressure at rupture was recorded as the bursting pressure (Fig. 1).

\section{Results}

\section{Macroscopic findings}

No aneurysm was produced by application of elastase to the adventitia of the arterial wall, regardless of enzyme concentrations. Various stages of aneurysm were induced by application of elastase to adventitia-dissected areas, ranging from spindleshaped enlargement of the whole vessel to localized protrusion followed by rupture. The observed stages correlated well with the gradual decrease in enzyme concentrations. Application of elastase 1:1 solution resulted in localized protrusion on the site of adventitia dissection, from which blood initially seeped. Rupture then occurred within 30 minutes in all six animals. The elastase 1:2 solution also caused protrusion of the adventitia-dissected region and oozing in all animals, but subsequent rupture was observed in only three of six animals. The elastase 1:4 to 1:16 solutions caused spindle-like distention from the adventitia-dissected region of the entire artery, and the common carotid artery kinked at the lesion. Some of these lesions showed blood oozing. However, no rupture developed within 2 hours of application. Only localized dilatations were seen

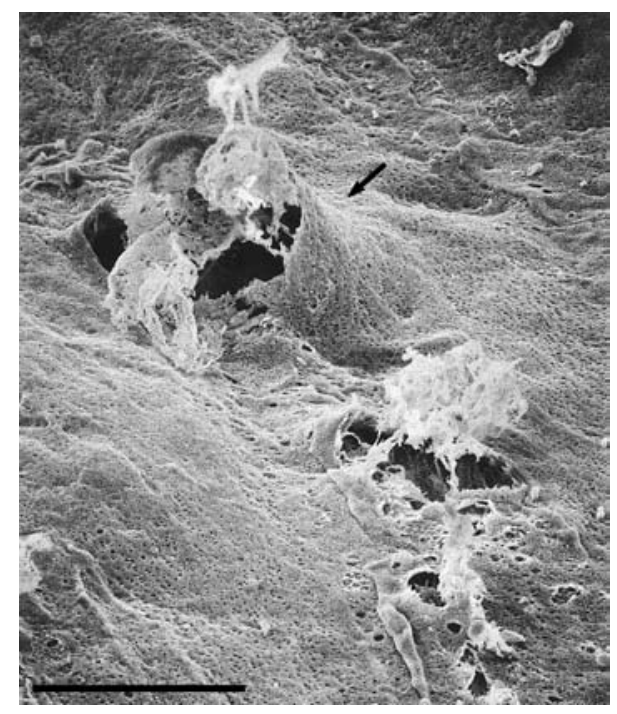

Fig. 4 Scanning electron micrograph of an experimental aneurysm created by application of elastase 1:32 solution. The endothelial damage in the area of the aneurysmal dilatation consists of crater-like vacuolization and focal areas of cellular desquamation with platelet attachment and formation of microthrombi (arrow). Bar $=\mathbf{3 . 0} \mu \mathrm{m}$.

with elastase solutions of 1:32 or weaker. These findings are summarized in Table 1 and Fig. 2.

\section{Histological findings}

Application of elastase 1:1 or 1:2 solution caused serious destruction of the internal elastic lamina and the media at the adventitia-dissected areas. Application of elastase 1:4 or more dilute solutions caused destruction and thinning of the internal elastic lamina, medial elastic fiber, and smooth muscle cell layer over the entire surface of the vessel. This destructive lesion became more localized and less invasive at more dilute elastase concentrations (Fig. 3). Scanning electron microscopy of the lesion caused by elastase 1:2 solution demonstrated structureless media, fibrin thrombi, and infiltration of leukocytes into the tunica intima. Similar findings, although less prominent, were caused by elastase 1:32 solution (Fig. 4).

\section{Forced rupture tests}

The bursting pressure of the aneurysms increased with the application of decreasing concentrations of elastase. The aneurysms produced by the elastase 1:2 solution ruptured at a blood pressure of less than $150 \mathrm{mmHg}$, whereas aneurysms induced by elastase $1: 4$ or more dilute solutions ruptured at around $500 \mathrm{mmHg}$, outside the physiological range 


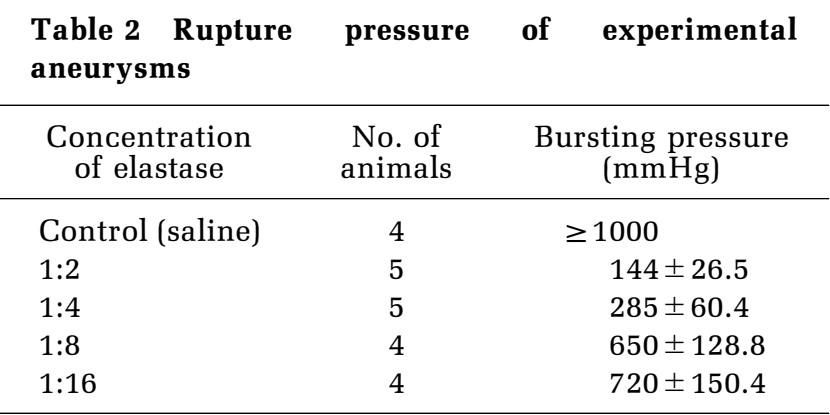

(Table 2). The bursting pressure of vessels with excised adventitia was $1000 \mathrm{mmHg}$ or more, but the maximal limit pressure could not be determined in our experimental system.

\section{Discussion}

The present study improved the previous aneurysm model, ${ }^{18)}$ in particular the limitations in reproducibility of aneurysm rupture. Specifically, the required concentrations of elastase were established to develop and rupture the aneurysm easily and with greater certainty.

Elastase can be applied from the intimal side $\mathrm{e}^{1,3,6,19)}$ or the adventitial side. ${ }^{12,18)}$ The separation of vessels is necessary to apply elastase to the tunica intima after converting a blood vessel, which is a complicated procedure and cannot be applied to living animals. ${ }^{1,19)}$ The enzyme may also be applied to the lumen after blocking the blood flow, which results in spindle-shaped extension of the vessel because localized application is impossible. ${ }^{3,6)}$ The present model $^{18)}$ applies elastase from the adventitial side, which facilitates manipulation. However, elastase applied over the adventitia did not induce an aneurysm of the lateral wall of the common carotid artery, possibly because the enzyme could not reach the media due to the external elastic lamina covering the thick adventitia in the carotid artery of the rabbit. ${ }^{18)}$ Aneurysms can be developed at the thyroid arterial bifurcation, where the arterial wall thins, but not at the common carotid arterial bifurcation, suggesting that the development of aneurysms is dependent on the thickness of the adventitia. ${ }^{18)} \mathrm{We}$ found that a small bulging of the vessel wall was consistently induced by partial dissection of the tunica adventitia before elastase application. Previous experiments showed that external mural excision of the adventitia of the common carotid artery developed aneurysmal lesions in two thirds of rats, but selective removal of the media failed to develop aneurysmal lesions in the chronic stage. ${ }^{29)}$

Elastase produces aneurysms, but collagenase causes the rupture of blood vessels without protrusion. ${ }^{8)}$ Therefore, collagenase is essential for injuring the adventitia of the arterial wall whereas only elastase is not sufficient. These findings suggest that the tunica adventitia is important in maintaining the strength of the vascular wall, and destruction of the internal elastic lamina and smooth muscle layer is essential to the development and growth of aneurysms. We demonstrated that dissection of the adventitia before application allowed the elastase to penetrate into the tunica media and then into the tunica intima, resulting in lysis of the internal elastic lamina. Consequently, blood vessels lost their normal morphology in both the longitudinal and transverse directions, and became locally swollen and serpiginous. The various stages of these pathological changes culminating in rupture of the lesion were produced by different concentrations of elastase. High concentrations of elastase caused the arterial wall to thin rapidly and protrude locally, and then blood oozing led to rupture. Low concentrations of elastase also induced protrusion of the arterial wall and local oozing, but not rupture.

Histological examination of these aneurysms demonstrated acute inflammatory reactions, including cellular infiltrate consisting of polymorphonuclear leukocytes and necrosis, which were presumably related to the enzymatic destruction of mural elements and subsequent aneurysmal dilatation. These findings differ from the essential histological features of natural aneurysms, such as abrupt termination of the tunica media and internal elastic lamina at the margin of the saccular orifice, as well as absence of inflammatory reaction. Therefore, this aneurysm model is considered to result from trauma through a proteolytic process.

Our forced rupture study revealed that thin and fragmented arterial walls could tolerate an intraarterial pressure up to $500 \mathrm{mmHg}$. However, a twofold increase in elastase solution concentration consistently induced aneurysm rupture. This experimental result suggests that the aneurysm wall is digested by various proteases, which leads to the final rupture, rather than thinning of the aneurysm wall as the arterial pressure mounts and rupture like a bulging balloon. This interpretation is consistent with the findings that elastase and various matrix metalloproteinases increase in the plasma and the aneurysm wall of patients., ${ }^{46)}$ Deficiency of $\alpha 1$ antitrypsin, an inhibitor of these enzymes, is also reported..$^{1021)}$ The matrix metalloproteinases found in the aneurysm wall possess both elastase and collagenase activities.,2,9,16,22) Collagenase activity may be necessary for the damage to the adventitia of the arterial wall that ultimately leads to rupture, 
whereas elastase may digest the arterial wall, causing a balloon-like dilatation.

The aneurysm model established in this study has the advantages of easy and rapid preparation, and can accurately control aneurysm development and rupture. Thus, the model is useful for the assessment of therapeutic efficacy of wrapping and coating materials.

\section{References}

1) Anidjar S, Salzmann J-L, Gentric D, Lagneau P, Camilleri J-P, Michel J-B: Elastase-induced experimental aneurysms in rats. Circulation 82: 973-981, 1990

2) Bruno G, Todor R, Lewis I, Chyatte D: Vascular extracellular matrix remodeling in cerebral aneurysms. J Neurosurg 89: 431-440, 1998

3) Cawly CM, Dawson RC, Shengelaia G, Bonner G, Barrow DL, Colohan ART: Arterial saccular aneurysm model in the rabbit. AJNR Am J Neuroradiol 17: 1761-1766, 1996

4) Chyatte D, Lewis I: Gelatinase activity and the occurrence of cerebral aneurysms. Stroke 28: 799-804, 1997

5) Chyatte D, Reilly J, Tilson MD: Morphometric analysis of reticular and elastin fibers in the cerebral arteries of patients with intracranial aneurysms. Neurosurgery 26: 939-943, 1990

6) Cloft HJ, Altes TA, Marx WF, Raible RJ, Hudson SB, Helm GA, Mandell JW, Jensen ME, Dion JE, Kallmes DF: Endovascular creation of an in vivo bifurcation aneurysm model in rabbits. Radiology 213: 223-228, 1999

7) Crompton MR: Mechanism of growth and rupture in cerebral berry aneurysms. Br Med J 5496: 1138-1142, 1966

8) Dobrin PB, Baker WH, Gley WC: Elastolytic and collagenolytic studies of arteries. Arch Surg 119: 405-409, 1984

9) Freestone T, Turner RJ, Coady A, Higman DJ, Greenhalgh RM, Powell JT: Inflammation and matrix metalloproteinases in the enlarging abdominal aortic aneurysm. Arterioscler Thromb Vasc Biol 15: 1145-1151, 1995

10) Gaetani P, Tartara F, Tancioni F, Klersy C, Forlino A, Baena RR: Activity of $\alpha 1$-antitrypsin and cigarette smoking in subarachnoid haemorrhage from ruptured aneurysm. J Neurol Sci 141: 33-38, 1996

11) German WJ, Black SPW: Experimental production of carotid aneurysms. N Engl J Med 250: 104-106, 1954

12) Gertz SD, Kurgan A, Eisenberg D: Aneurysm of the rabbit common carotid artery induced by periarterial application of calcium chloride in vivo. J Clin Invest 81: 649-656, 1988

13) Hashimoto N, Handa H, Hazama F: Experimentally induced cerebral aneurysms in rats. Surg Neurol 10: 3-8, 1978

14) Hashimoto N, Handa H, Nagata I, Hazama F: Animal models of cerebral aneurysms: Pathology and pathogenesis of induced cerebral aneurysms in rats. Neurol Res 6: 33-40, 1984

15) Hashimoto N, Kim C, Kikuchi H, Kojima M, Kang Y, Hazama F: Experimental induction of cerebral aneurysms in monkeys. J Neurosurg 67: 903-905, 1987

16) Kim SC, Singh M, Huang J, Prestigiacomo CJ, Winfree CJ, Solomon RA, Connolly ES: Matrix metalloproteinase-9 in cerebral aneurysms. Neurosurgery 41: 642-647, 1997

17) Klein SL, Israel JE, Kronengold RT: New burst test method for comparing strengths of blood vessel repairs. Microsurgery 16: 118-121, 1995

18) Miskolczi L, Guterman LR, Flaherty JD, Hopkins LN: Saccular aneurysm induction by elastase digestion of the arterial wall: A new animal model. Neurosurgery 43: 595-601, 1998

19) Miskolczi L, Guterman LR, Flaherty JD, Szikora I, Hopkins LN: Rapid saccular aneurysm induction by elastase application in vitro. Neurosurgery 41: 220-229, 1997

20) Nakagawa T, Hashi K: The incidence and treatment of asymptomatic unruptured cerebral aneurysms. J Neurosurg 80: 217-223, 1994

21) Schievink WI, Katzmann JA, Piepgras DG, Schaid DJ: Alpha-1-antitrypsin phenotypes among patients with intracranial aneurysms. J Neurosurg 84: 781-784, 1996

22) Skigrudas M, Awad IA, Kim J, Rothbart D, Crisculo G: Expression of angiogenesis factors and selected vascular wall matrix proteins in intracranial saccular aneurysms. Neurosurgery 39: 537-547, 1996

23) Stehbens WE: Experimental production of aneurysms by microvascular surgery in rabbits. Vasc Surg 7: 165-175, 1973

24) Suga S, Kawase T: [Strategy for unruptured intracranial aneurysms and rupture rate of previously unruptured aneurysms in Japan]. Surgery for Cerebral Stroke 29: 160-165, 2001 (Jpn)

25) Suzuki J, Ohara H: Clinicopathological study of cerebral aneurysms. Origin, rupture, repair, and growth. J Neurosurg 48: 505-514, 1978

26) Todor DR, Lewis I, Bruno G, Chyatte D: Identification of a serum gelatinase associated with the occurrence of cerebral aneurysms as pro-matrix metalloproteinase-2. Stroke 29: 1580-1583, 1998

27) Ujiie H, Tamano Y, Sasaki K, Hori T: Is the aspect ratio a reliable index for predicting the rupture of a saccular aneurysm? Neurosurgery 48: 495-503, 2001

28) Weir B: Unruptured aneurysms: a review. J Neurosurg 96: 3-42, 2002

29) Young PH, Fischer VW, Guity A, Young PA: Mural repair following obliteration of aneurysms: production of experimental aneurysms. Microsurgery 8: 128-137, 1987 
Address reprint requests to: H. Ujiie, M.D., Department of Neurosurgery, Neurological Institute, Tokyo Women's Medical University, 8-1 Kawada-cho, Shinjuku-ku, Tokyo 162-8666, Japan.

e-mail: huji@nij.twmu.ac.jp

\section{Commentary}

Modeling is a powerful tool in science and engineering, to enhance our abilities to explain, predict or modify specific phenomena. Modeling is as good, or as bad, as the inherent assumptions. It can test hypotheses, but these hypotheses are not generalizeable to the clinical situation without further testing of the validity and consistency of the model's assumptions.

Aneurysm modeling has been largely "misunderstood," as clinicians have thought of a unifying model replicating all relevant facets of the disease. This goal remains elusive, but a number of models have provided unique insights on lesion pathogenesis, others have allowed the study of hemodynamics and shear stresses, while other models have been more relevant to the training and simulation for endovascular therapy.

The current model described by Sasaki and colleagues allows the study of another dimension of these lesions: acute aneurysmal dilation caused by elastase digestion of the vessel wall matrix. An attractive feature of the model is its high success rate and the optimized conditions for simulated aneurysm growth and rupture. The realistic aneurysm size will allow applications for optimizing molecular imaging of aneurysm wall in animals before use in humans. The model is also attractive for testing therapeutic factors that may alter resilience or vulnerability to aneurysm growth or rupture.

But alas, this model does not reflect the unique features of cerebral vessels, and the time course of aneurysm development and rupture is compressed into an unrealistically short time window. This model, as all others, does not answer all questions about aneurysms. And as with other models, any hypothesis about applicability or generalizeability of observations with this model to real aneurysms will need its own testing and validation.

Issam A. AwAD, M.D., M.Sc., F.A.C.S., M.A. (Hon.) Professor of Neurosurgery Evanston Northwestern Healthcare Northwestern University Feinberg School of Medicine Evanston, Illinois, U.S.A.

Sakai et al. present a new approach to produce an animal model of aneurysm through the application of elastase. Recently, many reports have indicated that degradation of arterial wall by proteases is an essential step in aneurysm formation, and elastase and collagenase are especially important for such vascular remodeling. The authors focused on the application of elastase and analyzed its concentrations for reproducibility of aneurysm development and rupture. Their animal model could be produced easily and certainly. These features support the hypothesis that this model should be suitable for studying the efficacy of newly artificial materials for wrapping and coating.

Previous reports showed the aneurysmal wall mainly consisted of degenerated adventitia, resulting from thinning of the smooth muscle cell layer and disappearance of the internal elastic lamina. In this model, the authors dissected the tunica adventitia before application of elastase. As documented, this model was truly a traumatic model by direct manipulation, and its histological features were not the same as the actual aneurysm. This is why this model has limitations in the analysis of the pathogenesis of aneurysms.

However, the force-rupture study of this model revealed that increases in elastase solution concentrations induced aneurysm rupture. This fact suggests that the arterial wall was degraded by various proteases in the process of aneurysm formation. Regarding this point, vascular remodeling of the lesion seemed to be somewhat similar to human aneurysm formation.

Susumu MiYamoto, M.D. Department of Neurosurgery National Cardiovascular Center Suita, Osaka, Japan

The authors present a new animal model to induce aneurysm formation in the common carotid artery by the topical application of elastase following removal of the adventitia. Regarding experimental aneurysm models, Hashimoto et al. ${ }^{2)}$ have developed a model closely resembling human cerebral aneurysm formation. However, the location of aneurysmal growth is unpredictable and it takes long time for the induction of aneurysms. To create the aneurysm more rapidly and consistently at the desired location, Miskolczi et al. ${ }^{3)}$ have developed a new model by the topical application of elastase to arterial branch points. With this model, they suggested important roles of elastase in the cerebral arterial wall for the aneurysm formation. Anidjar et al. ${ }^{1)}$ have demonstrated a close relationship between aneurysm formation and elastolytic activity in the arterial wall. These reports indicate that the experimental model by the topical application of elastase serves as a good model for 
aneurysm research. In addition to the topical application of elastase, the authors decreased the pressure threshold of the arterial wall by removing the adventitia to induce aneurysms more consistently than in the Miskolczi's model. This model is basically a traumatic model and serves as a model for clarifying the mechanism of traumatic aneurysm formation or for evaluating the therapeutic efficacy of wrapping and coating materials. The authors have thus developed a new rabbit model of aneurysm formation. However, it is desirable to evaluate the histological changes more precisely and/or to quantify the elastolytic activity within the arterial wall.

\section{References}

1) Anidjar S, Salzmann JL, Gentric $D$, Lagneau $P$, Camilleri JP, Michel JG: Elastase-induced experimental aneurysms in rats. Circulation 82: 973-981, 1990

2) Hashimoto N, Kim C, Kikuchi H, Kojima M, Kang Y, Hazama F: Experimental induction of cerebral aneurysms in monkeys. J Neurosurg 67: 903-905, 1987

3) Miskolczi L, Guterman LR, Flaherty JD, Hopkins LN: Saccular aneurysm induction by elastase digestion of the arterial wall: a new animal model. Neurosurgery 43: 595-601, 1998

Tomio SASAKI, M.D. Department of Neurosurgery Kyushu University School of Medicine Fukuoka, Japan 